\title{
Gallbladder Papillary Neoplasm with Low Grade Intraepithelial Neoplasia
}

National Cancer Institute

\section{Source}

National Cancer Institute. Gallbladder Papillary Neoplasm with Low Grade Intraepithelial Neoplasia. NCI Thesaurus. Code C96877.

A non-invasive, papillary epithelial neoplasm that arises from the epithelium of the gallbladder. It is characterized by the presence of mild epithelial atypia. 